\title{
Behavioral Modeling and Digital Predistortion of $2 \times 2$ MIMO Wireless Transmitters with Enhanced Multivariable Polynomials
}

\author{
Imene Zemzemi \\ Department of Physics, \\ University of Tunis El Manar, \\ Tunis, Tunisia \\ imizemzmi@gmail.com
}

\author{
Noureddine Boulejfen \\ Research Centre for Microelectronics and Nanotechnology \\ Technopole of Sousse, \\ Sousse, Tunisia \\ nboulejf@ieee.org
}

\begin{abstract}
In this paper we propose a novel concept named Enhanced Multivariable Memory Polynomials (EMVP) for the behavioral modeling and linearization of Multiple Input Multiple Output (MIMO) transmitters in the presence of linear (LC) and nonlinear (NLC) coupling effects. The proposed model improves the linearization performance of the conventional Multivariable Memory Polynomials (MVP) model. Its performance is experimentally compared with the ones of three popular polynomial models. EMVP model has succeeded in offering better linearization of MIMO transmitters, with fewer coefficients, compared to the conventional models. Experimental results showed improvement in Adjacent Channel Power Ratio $(A C P R<-60 d B c)$ and Normalized Mean Square Error (NMSE $<-45 \mathrm{~dB})$ for LTE signal excitation with $10 \mathrm{MHz}$ bandwidth, in the presence of both linear and nonlinear couplings of $20 \mathrm{~dB}$.
\end{abstract}

Keywords-behavioral modeling;Digital Pre-Distortion (DPD); Multiple-Input Multiple-Output (MIMO); Enhanced Multivariable Memory Polynomials (EMVP)

\section{INTRODUCTION}

In $5 \mathrm{G}$ wireless communication systems, billions of connections could exist concurrently for various data transmission types $[1,2]$. MIMO transmitters are considered as a feasible solution to fulfill these high requirements [3-15]. MIMO systems deal with simultaneous transmission and reception of multiple signals, through the same radio channel and frequency bandwidth, by exploiting multipath propagation. [6-12]. Moreover, MIMO systems have been developed to handle concurrent dual-band signals [13-17]. Despite their efficiency in terms of data transmission rates, MIMO transmitters suffer from several problems. On top of the nonlinearity and memory effects, inherited from single inputsingle output (SISO) transmitters, MIMO systems suffer from the coupling between adjacent amplification branches. This crosstalk [8] becomes stronger in MMIC implementation of MIMO transmitters. In the published literature crosstalk is classified as linear (LC) and nonlinear (NLC) [9-11]. In [620], digital predistorters (DPD) have been used to reduce the distortion effects of MIMO systems. In [9], the crossover model (MCR) is based on the summation of two nonlinear
SISO models. This model has the advantage of low amount of coefficients, but its performance degrades in the presence of strong crosstalk (NLC and/or LC of levels over -30dB). In [10], a robust model based on Multivariable Polynomials (MVP) has been presented. This model is capable of handling memory effects, as well as strong linear and nonlinear crosstalk, with a cost of a large amount of coefficients and high complexity. In [11], a curtailed version of the MVP based model (CMVP) has been developed through the elimination of certain terms, by considering only the nonlinear crosstalk. This model, and its corresponding DPD, is able to reach almost the same performance as the MVP based model with less coefficients. Therefore, MVP and CMVP models are assumed as good references for measuring the performance of the proposed model. In this paper, we propose a robust polynomial model for the digital predistortion of MIMO systems, named as the Enhanced Multivariable Polynomials (EMVP) model. This new model is based on the enhancement of the MVP model's efficiency by adding properly selected intermodulation and cross terms.

\section{POLYNOMIAL MODELING OF MIMO TRANSMITTERS}

In SISO transmission systems, RF power amplifiers are critical components in terms of energy consumption, nonlinearity and memory effects that cause signal distortion. MIMO power amplifiers inherit these effects, while exhibiting an additional coupling effect between amplification branches. In fact, input signals are transmitted at the same time and carrier frequency through transmitter paths. The resulting coupling depends on certain parameters, such as PA nonlinearity, power of input signals, and integration density which is exponentially increasing in modern communication systems like $4 \mathrm{G}$ and $5 \mathrm{G}$ networks. To compensate for coupling, PA nonlinearity and memory effects, a 2D DPD with reasonable complexity and performance compromise is required [3]. Figure 1 shows the proposed diagram of a $2 \times 2$ MIMO DPD system, based on the indirect learning architecture where $u_{i}(n), y_{i}(n)$ and $z_{i}(n)$ represent the baseband input signal, the PA output signal and the predistorted signal of the amplification branch $i$ respectively. Crosstalk is caused by the coupling between different transmitter's paths or due to the 
leakage through the common LO, classified LC and NLC. NLC occurs at the PA input path while LC occurs at the PA output.

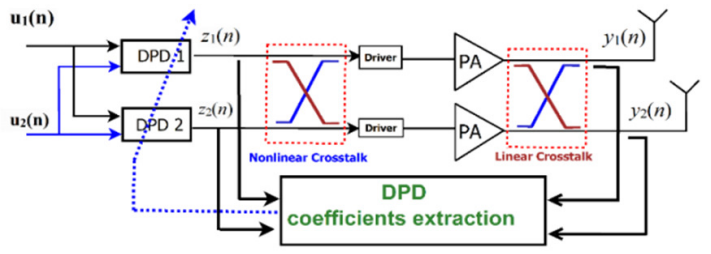

Fig. 1. Architecture of a $2 \times 2$ MIMO transmitter with DPD

Two coupling scenarios will be considered in order to properly assess the performance of the proposed DPD. It can be expected that performance will degrade when crosstalk at input is stronger than $20 \mathrm{~dB}$. This is expected to occur for high integration density, foreseen in $4 \mathrm{G}$ systems and beyond [3]. For comparison purposes, two recently published DPDs, based on multivariable polynomial models, will be simulated.

\section{A. Multivariable Memory Polynomial Model (MVP)}

In order to enhance model's accuracy for MIMO transmitters with strong crosstalk, an inclusive MIMO polynomial model has been proposed in [10]. The output of each amplification branch is modeled considering only NLC MIMO transmitter's input. The output of the MVP $2 \times 2$ MIMO model can be written as:

$$
\begin{gathered}
y^{(i)}(n)=\sum_{m=0}^{M} \sum_{k=0}^{K-1} \sum_{q=0}^{k} b_{k, q, m}^{i .1} u_{1}(j)\left|u_{1}(j)\right|^{(k-q)}\left|u_{2}(j)\right|^{q} \\
+\sum_{m=0}^{M} \sum_{k=0}^{K-1} \sum_{q=0}^{k} b_{k, q, m}^{i .2} u_{2}(j)\left|u_{2}(j)\right|^{(k-q)}\left|u_{1}(j)\right|^{q}
\end{gathered}
$$

where $K$ is the nonlinearity order, $M$ is the memory depth, $b_{k, q, m}^{(i, 1)}$ and $b_{k, q, m}^{(i, 2)}$ are the coefficients of each model's branches, $u_{1}(n)$ and $u_{2}(n)$ are input signals and $y_{M V P}^{(i)}(n)$ is the $i^{\text {th }}$ output of the MIMO transmitter, with $i=\{1,2\}$. MVP model has a relatively high number of coefficients and is expected to exhibit good performance.

\section{B. Curtailed Multivariable Memory Polynomial Model (CMVP)} by:

The output $y^{(i)}$ of the $2 \times 2$ CMVP based model is given

$$
\begin{aligned}
& y^{(i)}(n)=\sum_{m=0}^{M} \sum_{k=0}^{K-1} b_{k, m}^{i .1} u_{1}(j)\left|u_{1}(j)\right|^{k} \\
& +\sum_{m=0}^{M} \sum_{k=0}^{K / 2-1} b_{k, m}^{i .2} u_{2}(j)\left|u_{2}(j)\right|^{k} \\
& +\sum_{m=0}^{M} \sum_{k=1}^{K / 2-1} \sum_{q=1}^{k} b_{k, q, m}^{i .3} u_{1}(j)\left|u_{1}(j)\right|^{(k-q)}\left|u_{2}(j)\right|^{q}
\end{aligned}
$$

with $u_{1}(n)$ and $u_{2}(n)$ being the input signals, $K$ the non-linearity order, $M$ the memory depth, $b_{k, m}^{i .1}, b_{k, m}^{i .2}$ and $b_{k, q, m}^{i .3}$ the model coefficients, and $y^{(i)}(n)$ the output of the amplification branch $i$. The complexity of CMVP based model is way less than MVP, with comparable performance.

\section{Proposed Model (EMVP)}

MIMO transmitters' model aims to predict precisely PA nonlinearity and memory effects, as well as LC and NLC between PA branches. Hence, the model's complexity is increasing with the strength of these undesired effects. A compromise between accuracy and complexity is very helpful on implementing any model. In this context, we propose EMVP MIMO model that eliminates the even nonlinear terms, while adding all cross terms between input signals and intermodulation terms of the main transmitted channels. Thus, the output of the proposed model, including both LC and NLC, can be expressed as:

$$
y^{(i)}(n)=y_{L}^{(i)}(n)+y_{N L}^{(i)}(n)
$$

where $y_{N L}^{(i)}(n)$ is the output component with cross terms that model the non-linear crosstalk, given by:

$$
\begin{gathered}
y_{N L}^{(i)}(n)=\sum_{m=0}^{M} u_{1}(j) \sum_{k=0}^{K-1} \sum_{q=0}^{k} b_{k, q, m}^{i .1}\left|u_{1}(j)\right|^{2(k-q)}\left|u_{2}(j)\right|^{2 q} \\
+\sum_{m=0}^{M} u_{2}(j) \sum_{k=0}^{K-1} \sum_{q=0}^{k} b_{k, q, m}^{i .2}\left|u_{2}(j)\right|^{2(k-q)}\left|u_{1}(j)\right|^{2 q}
\end{gathered}
$$

However, $y_{L}^{(i)}$ predicts LC, which, for a $2 \times 2$ MIMO transmitter in the presence of $\mathrm{LC}$, can be given by:

$$
\begin{gathered}
y_{L}^{(i)}(n)=\sum_{m=0}^{M} u_{1}(j) \Delta_{1}\left(u_{1}(j), u_{2}(j)\right) \sum_{k=1}^{K-1} b_{k, m}^{i .3}\left|u_{1}(j)\right|^{2 k-2} \\
+\sum_{m=0}^{M} u_{2}(j) \Delta\left(u_{2}(j), u_{1}(j)\right) \sum_{k=1}^{K-1} b_{k, m}^{i .4}\left|u_{2}(j)\right|^{2 k-2}
\end{gathered}
$$

with $\Delta\left(u_{1}(j), u_{2}(j)\right)=u_{1}(j) u_{2}^{*}(j)+u_{2}(j) u_{1}^{*}(j),\left({ }^{*}\right)$ being the conjugate operator, $j=n-m$, is the output of channel $1, u_{1}(n)$ and $u_{2}(n)$ are the input signals, $K$ the nonlinearity order defined as (nonlinearity degree +1$) / 2$ and $M$ is the memory depth. Thus, the output of a transmitter path can be expressed as:

$$
\begin{gathered}
y^{(i)}(n)=\sum_{m=0}^{M} u_{1}(j) \sum_{k=0}^{K-1} \sum_{q=0}^{k} b_{k, q, m}^{i .1}\left|u_{1}(j)\right|^{2(k-q)}\left|u_{2}(j)\right|^{2 q} \\
+\sum_{m=0}^{M} u_{2}(j) \sum_{k=0}^{K-1} \sum_{q=0}^{k} b_{k, q, m}^{i .2}\left|u_{2}(j)\right|^{2(k-q)}\left|u_{1}(j)\right|^{2 q} \\
+\sum_{m=0}^{M} u_{1}(j) \Delta_{1}\left(u_{1}(j), u_{2}(j)\right) \sum_{k=1}^{K-1} b_{k, m}^{i .3}\left|u_{1}(j)\right|^{2 k-2} \\
+\sum_{m=0}^{M} u_{2}(j) \Delta\left(u_{2}(j), u_{1}(j)\right) \sum_{k=1}^{K-1} b_{k, m}^{i .4}\left|u_{2}(j)\right|^{2 k-2}
\end{gathered}
$$

where $b_{p, q, m}^{i .1}, b_{p, m}^{i .3}, b_{p, q, m}^{i .2}$ and $b_{p, m}^{i .4}$ are model's coefficients. EMVP model includes all cross terms and intermodulation terms of signals in transmitter's output. We added in (2) two terms for each nonlinearity order $K$. The model is expected to perform well with limited memory depths, as it will be demonstrated below in experimental validation. Table I presents the expressions that calculate the number of coefficients for the three considered models. Figure 2(a) shows the variation of these numbers as a function of the model's nonlinearity order for a constant memory depth $M=3$. Figure 2(a) reveals that the amount of MVP model's coefficients 
augments exponentially with nonlinearity order, as compared to CMVP and the proposed EMVP models that exhibit comparable behavior.

TABLE I. NUMBER OF COEFFICIENTS FOR $2 \times 2$ MIMO MODELS

\begin{tabular}{|c|c|}
\hline Model & Number of Coefficients \\
\hline CMVP & $2(M+1)\left(K+\right.$ floor $\left.(K / 2) * \frac{\text { floor }(K / 2)}{2}\right)$ \\
\hline MVP & $K(K+1)(M+1)$ \\
\hline EMVP & $K M+\frac{((K+3)(K+1)(M+1))}{4}-(M+1)$ \\
\hline
\end{tabular}

\section{Models' Identification}

Model's identification is a critical procedure. In fact, model's coefficients need to be carefully estimated in order to reach the highest performance on any given behavioural model. There are different parameter identification strategies such as the Least Squares (LS) algorithm, which is widely used to identify polynomial models with linear parameters. However, iterative procedures such as Recursive Least Squares (RLS) can be used for models with no linear parameters. In order to use the LS identification procedure expressions in (1), (2) and (4) are reformulated into matrix form as:

$$
\mathrm{Y}^{(\mathrm{i})}=\mathrm{B}^{(\mathrm{i})} \mathrm{C}^{(\mathrm{i})}
$$

where $Y^{(i)}$ is the $N \times 1$ vector representing samples of the $i^{\text {th }} \mathrm{PA}$ output and $B^{(i)}$ is the $N \times S$ data matrix, relative to branch $i$, that includes nonlinear input terms obtained from model's input. $C^{(i)}$ is an $S \times 1$ vector containing model coefficients for branch $i$, where $S$ is the overall model size. For simplicity, $i$ subscript will be dropped in subsequent equations. Data matrices for the models are given below:

$$
\begin{aligned}
& \mathrm{B}_{E M V P}^{(1)}=\left[\begin{array}{llll}
\mathrm{B}_{E M V P}^{(1.1)} & \mathrm{B}_{E M V P}^{(1.2)} & \mathrm{B}_{E M V P}^{(1.3)} & \mathrm{B}_{E M V P}^{(1.4)}
\end{array}\right] \\
& \mathrm{B}_{M V P}^{(1)}=\left[\begin{array}{ll}
\mathrm{B}_{M V P}^{(1.1)} & \mathrm{B}_{M V P}^{(1.2)}
\end{array}\right] \\
& \mathrm{B}_{C M V P}^{(1)}=\left[\begin{array}{ll}
\mathrm{B}_{C M V P}^{(1.1)} & \mathrm{B}_{C M V P}^{(1.2)} \mathrm{B}_{C M V P}^{(1.3)}
\end{array}\right]
\end{aligned}
$$

where each row of the component matrices (corresponding to one time index $j=n-m$ ) is given by:

$$
\begin{aligned}
& \mathrm{B}_{M V P}^{(1.1)}(n)=\left[u_{1}(j) \ldots u_{1}(j)\left|u_{1}(j)\right|^{(k-q)}\left|u_{2}(j)\right|^{q} \ldots u_{1}(j)\left|u_{2}(j)\right|^{(K-1)}\right] \\
& \mathrm{B}_{\text {MVP }}^{(1.2)}(n)=\left[u_{2}(j) \ldots u_{2}(j)\left|u_{2}(j)\right|^{(k-q)}\left|u_{1}(j)\right|^{q} \ldots u_{2}(j)\left|u_{1}(j)\right|^{(K-1)}\right] \\
& \mathrm{B}_{\text {CMVP }}^{(1.1)}(n)=\left[u_{1}(j) \ldots u_{1}(j)\left|u_{1}(j)\right|^{k} \ldots u_{1}(j)\left|u_{1}(j)\right|^{(K-1)}\right] \\
& \mathrm{B}_{\text {CMVP }}^{(1.2)}(n)=\left[u_{2}(j) \ldots u_{2}(j)\left|u_{2}(j)\right|^{k} \ldots u_{2}(j)\left|u_{2}(j)\right|^{(K / 2-1)}\right] \\
& \mathrm{B}_{\text {CMVP }}^{(1.3)}(n)=\left[u_{1}(j) \ldots u_{1}(j)\left|u_{1}(j)\right|^{(k-q)}\left|u_{2}(j)\right|^{q} \ldots u_{1}(j)\left|u_{2}(j)\right|^{(K / 2-1)}\right] \\
& \mathrm{B}_{E M V P}^{(1.1)}(n)=\left[\begin{array}{ll}
u_{1}(j) \ldots & u_{1}(j)\left|u_{1}(j)\right|^{2(k-q)}\left|u_{2}(j)\right|^{2 q} \\
\ldots & u_{1}(j)\left|u_{2}(j)\right|^{2(K-1)}
\end{array}\right]
\end{aligned}
$$

$$
\begin{aligned}
& \mathrm{B}_{E M V P}^{(1.2)}(n)=\left[\begin{array}{cc}
u_{2}(j) \ldots & u_{2}(j)\left|u_{2}(j)\right|^{2(k-q)}\left|u_{1}(j)\right|^{2 q} \\
\ldots & u_{2}(j)\left|u_{1}(j)\right|^{2(K-1)}
\end{array}\right] \\
& \mathrm{B}_{E M V P}^{(1.3)}(n)=\left[\begin{array}{ll}
u_{1}(j) \ldots & u_{1}(j)\left|u_{1}(j)\right|^{2(K-1)}
\end{array}\right] \Delta\left(u_{1}(j), u_{2}(j)\right) \\
& \mathrm{B}_{E M V P}^{(1.4)}(n)=\left[\begin{array}{lll}
u_{2}(j) \ldots & u_{2}(j)\left|u_{2}(j)\right|^{2(K-1)}
\end{array}\right] \Delta\left(u_{1}(j), u_{2}(j)\right)
\end{aligned}
$$

After constructing model's matrices, as outlined above, coefficients are typically extracted using LS [19] as:

$$
\hat{\mathrm{C}}_{L S}^{(i)}=\left(\mathrm{B}^{(i) \mathrm{H}} \mathrm{B}^{(i)}\right)^{-1} \mathrm{~B}^{(i) H} \mathrm{Y}^{(i)}
$$

$\hat{\mathrm{C}}_{L S}^{(i)}$ is obtained by the pseudo inverse of matrix $B^{(i)}$, with $H$ being the operator denoting the Hermitian transpose. LS method has been extensively used to extract parameters of behavioral models and DPDs. However, it is known to suffer from numerical stability issues due to the matrix inversion step when large ill-conditioned model matrices are involved.

\section{NUMERICAL STABILITY ANALYSIS}

Numerical stability of the model's identification procedure, using the LS algorithm, mainly relies on the conditioning of the observation matrix $\left(B^{(i) H} B^{(i)}\right)$. Inverting ill-conditioned matrix results in inaccurate and dispersive coefficients that reduce the performance of the corresponding DPD. This problem becomes more severe when implementing DPD in a fixed point calculation environment such as DSP and FPGA hardware. This would require large word length, which increases the cost of practical system realizations $\lceil 20\rceil$. A good metric for the matrix condition is the condition number is given by:

$$
\rho=\|\mathrm{B}\|_{2} \times\left\|\mathrm{B}^{-1}\right\|_{2}
$$

where $B$ is the square observation matrix to be inverted and \|\|$_{2}$ operator refers to the 2-norm. Figure 2(b) presents the condition number of the observation matrices for the three considered models as a function of the nonlinearity order with constant memory depth $\mathrm{M}=3$. The Figure shows that the EMVP model has the lowest condition number for all $\mathrm{K}$, which reveals the stability and the robustness of the proposed model compared to CMVP and MVP. Table II lists the condition numbers for the three models, for different memory depths. Once again, the proposed model has shown its superiority for all combinations of $(\mathrm{M}, \mathrm{K})$.

TABLE II. CONDITION NUMBER FOR THE $2 \times 2$ MIMO CASE

\begin{tabular}{|c|c|c|c|c|c|}
\hline $\begin{array}{c}\text { Memory } \\
\text { depths }\end{array}$ & \multirow{2}{*}{ Models } & \multicolumn{4}{|c|}{$\mathbf{K}$} \\
\cline { 3 - 6 } & & $\mathbf{1}$ & $\mathbf{5}$ & $\mathbf{9}$ & $\mathbf{1 1}$ \\
\hline \multirow{3}{*}{$\mathbf{M}=\mathbf{0}$} & MVP & 1.05 & $8.02 .10^{06}$ & $4.41 .10^{13}$ & $1.12 .10^{17}$ \\
\cline { 2 - 6 } & $\mathbf{C M V P}$ & 1.05 & $3.62 .10^{06}$ & $8.95 .10^{13}$ & $3.19 .10^{17}$ \\
\cline { 2 - 6 } & $\mathbf{E M V P}$ & 1.05 & 4479.2 & $1.53 .10^{07}$ & $1.08 .10^{09}$ \\
\hline \multirow{3}{*}{$\mathbf{M}=\mathbf{3}$} & MVP & $2.01 .10^{10}$ & $3.86 .10^{12}$ & $1.98 .10^{18}$ & $1.72 .10^{20}$ \\
\cline { 2 - 6 } & $\mathbf{C M V P}$ & $2.01 .10^{10}$ & $7.16 .10^{12}$ & $1.98 .10^{18}$ & $4.27 .10^{20}$ \\
\cline { 2 - 6 } & $\mathbf{E M V P}$ & $2.01 .10^{10}$ & $1.01 .10^{10}$ & $2.27 .10^{13}$ & $6.11 .10^{14}$ \\
\hline \multirow{3}{*}{$\mathbf{M}=\mathbf{5}$} & $\mathbf{M V P}$ & $8.38 .10^{15}$ & $1.07 .10^{17}$ & $2.93 .10^{20}$ & $3.77 .10^{21}$ \\
\cline { 2 - 6 } & $\mathbf{C M V P}$ & $8.38 .10^{15}$ & $8.42 .10^{17}$ & $3.41 .10^{20}$ & $7.79 .10^{21}$ \\
\cline { 2 - 6 } & $\mathbf{E M V P}$ & $8.38 .10^{15}$ & $1.06 .10^{16}$ & $9.78 .10^{16}$ & $1.31 .10^{18}$ \\
\hline
\end{tabular}


(a)

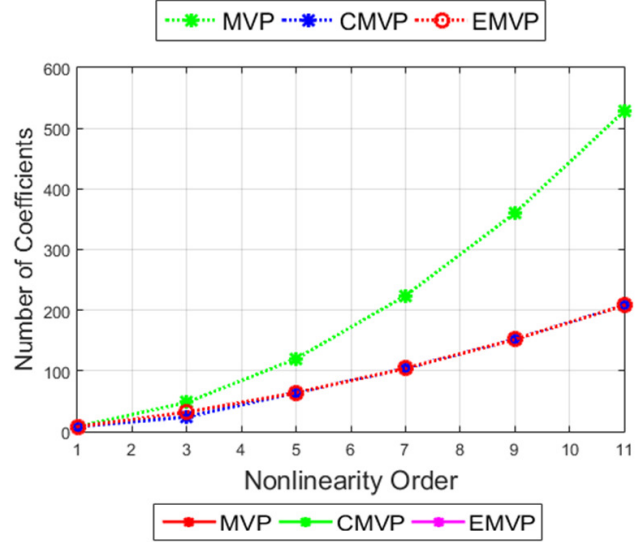

(b)

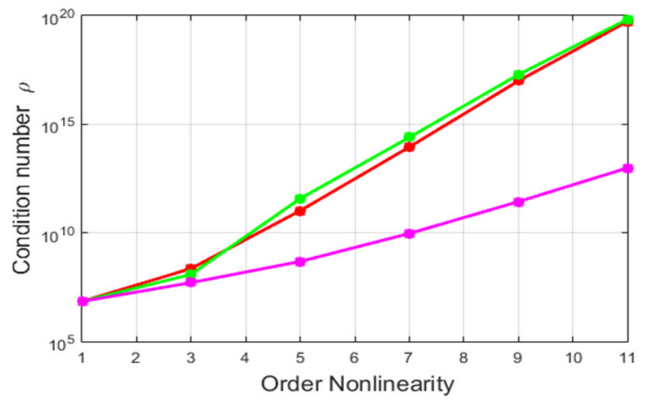

Fig. 2. (a) Number of coefficients as a function of the nonlinearity order K for $\mathrm{M}=3$, (b) Condition number as a function of $\mathrm{K}$ and $\mathrm{M}=3$.

\section{EXPERIMENTAL VALIDATION}

\section{A. Experimental Setup}

Experimental setup used for both DPD extraction and MIMO forward modeling is illustrated in Figure 3, where two complex baseband signals are generated and fed into two synchronized Rohde \& Schwarz SMBV100A vector signal generators, to produce the input RF signals with the same carrier. Two identical power amplifiers RF Mini-Circuits ZHL42 constitute the DUT setup, while two cross-connected couplers are used to produce a path of $20 \mathrm{~dB}$ coupling between the input signals. Couplers are placed in front of the PAs for nonlinear coupling and after them for a linear one. Input RF signals are independent LTE signals centered on $2.14 \mathrm{GHz}$ with $10 \mathrm{MHz}$ bandwidth, $-8 \mathrm{~dB}$ average power and $11 \mathrm{~dB}$ peak-toaverage power ratio (PAPR). MIMO system's RF outputs are converted to Intermediate Frequency (IF) signals. An ADQ214 analog-to-digital converter (ADC) samples IF signals with $400 \mathrm{MHz}$ sampling rate and 14 bit resolution. Indirect Learning Architecture (ILA) is used following the same methodology as in $[10,11]$.

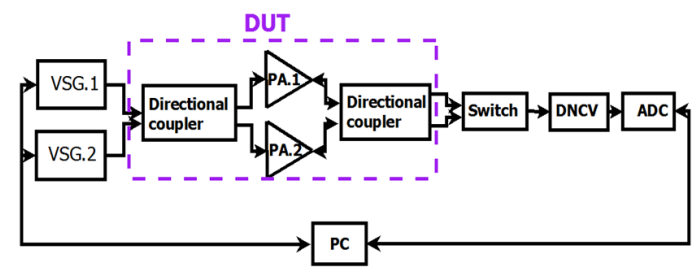

Fig. 3. Setup diagram

\section{B. Model and DPD Performance Metrics}

\section{1) Normalized Mean Square Error (NMSE)}

Time domain NMSE $[22,23]$ has been used in order to evaluate the accuracy of the proposed MIMO model in predicting transmitter output. NMSE is expressed as:

$$
N M S E_{d B}=10 \log _{10}\left[\frac{\sum_{n=1}^{N}\left|y_{m s}\left(n T_{S}\right)-y_{e s t}\left(n T_{S}\right)\right|^{2}}{\sum_{n=1}^{N}\left|y_{m s}\left(n T_{S}\right)\right|^{2}}\right]
$$

where $y_{m s}\left(n T_{s}\right)$ and $y_{\text {est }}\left(n T_{s}\right)$ are the measured and predicted outputs respectively. Typically, values below $-40 \mathrm{~dB}$ are desirable for NMSE [10-11].

\section{2) Adjacent Channel Power Ratio (ACPR)}

In order to study the linearization performance of a DPD, the ACPR metric is commonly used [22, 23]:

$$
A C P R_{d B}=\frac{\int_{\text {Adj.ch }}|Y(f)|^{2} d f}{\int_{\text {main.ch }}|Y(f)|^{2} d f}
$$

ACPR can be evaluated for the adjacent channels above and below the main frequency band - known as the upper and lower ACPR - in units of $\mathrm{dBc}$ ( $\mathrm{dB}$ below the carrier). $|Y(f)|^{2}$ is the power spectral density of the $y(n)$ output.

\section{Results}

The performance of the proposed EMVP model was evaluated against CMVP and MVP models, in terms of forward modeling and DPD. Coupling scenarios shown in Table III were considered for the evaluation procedure.

TABLE III. COUPLING SCENARIOS

\begin{tabular}{|c|c|c|}
\hline \multirow{2}{*}{ Scenarios } & \multicolumn{2}{|c|}{ Crosstalk levels (dB) } \\
\cline { 2 - 3 } & NLC & LC \\
\hline Scenario 1 & 20 & 0 \\
\hline Scenario 2 & 20 & 20 \\
\hline
\end{tabular}

\section{1) Forward Modeling}

Performance evaluation results of EMVP model using a MIMO transmitter are shown in Tables IV and V. NMSE and the amount of coefficients are measured for nonlinearity order $\mathrm{K}=11$. Table IV presents the NMSEs for the first scenario. Results reveal that the NMSE of the EMVP model is the lowest with an average of 3-4dB lower than MVP and CMVP. In addition, EMVP and CMVP have the same number of coefficients. However, EMVP model achieves maximum NMSE performance for a memory depth of $\mathrm{M}=3$, while MVP and CMVP reach their best NMSE performance for $\mathrm{M}=5$. Table $\mathrm{V}$ presents the NMSEs for the second scenario. Once again, the proposed model exhibits better performance, with an NMSE of $-45.28 \mathrm{~dB}$ compared to MVP and CMVP, which did not drop below $-42.10 \mathrm{~dB}$. In order to further investigate the models' convergence behavior and respective sensitivity, EMVP and CMVP were extracted while sweeping the value of 
$\mathrm{K}$ for memoryless $(\mathrm{M}=0)$ and memory models with $\mathrm{M}=3$. Obtained results for the two scenarios are shown in Figure 4. It is clear that EMVP can reach better NMSE performance $(-45 \mathrm{~dB})$ and offers the fastest convergence for a similar number of coefficients.

TABLE IV. NMSE FOR THE FIRST MEASUREMENT SCENARIO

\begin{tabular}{|c|c|c|c|c|}
\hline \multirow{2}{*}{ Models } & \multirow{2}{*}{ M } & \multirow{2}{*}{$\begin{array}{c}\text { Number of } \\
\text { coefficients }\end{array}$} & \multicolumn{2}{|c|}{ NMSE (dB) } \\
\cline { 4 - 5 } & & $\boldsymbol{y}^{(1)}$ & $\boldsymbol{y}^{(2)}$ \\
\hline \multirow{2}{*}{ MVP } & 3 & 528 & -44.05 & -43.99 \\
\cline { 2 - 5 } & 5 & 792 & -44.76 & -44.46 \\
\hline \multirow{2}{*}{ CMVP } & 3 & 208 & -43.05 & -43.15 \\
\cline { 2 - 5 } & 5 & 312 & -43.97 & -43.42 \\
\hline \multirow{2}{*}{ EMVP } & 3 & 208 & -46.93 & -47.05 \\
\cline { 2 - 5 } & 5 & 312 & -47.11 & -47.15 \\
\hline
\end{tabular}

TABLE V. NMSE FOR THE SECOND MEASUREMENT SCENARIO

\begin{tabular}{|c|c|c|c|c|}
\hline \multirow{2}{*}{ Models } & \multirow{2}{*}{$\mathbf{M}$} & \multirow{2}{*}{$\begin{array}{c}\text { Number of } \\
\text { coefficients }\end{array}$} & \multicolumn{2}{|c|}{ NMSE (dB) } \\
\cline { 4 - 5 } & & 528 & $\boldsymbol{y}^{(\text {() }}$ & $\boldsymbol{y}^{(2)}$ \\
\hline \multirow{2}{*}{ MVP } & 3 & 792 & -41.97 & -41.25 \\
\cline { 2 - 5 } & 5 & 208 & -42.10 & -41.96 \\
\hline \multirow{2}{*}{ CMVP } & 3 & 312 & -41.40 & -40.90 \\
\cline { 2 - 5 } & 5 & 208 & -45.28 & -41.33 \\
\hline \multirow{2}{*}{ EMVP } & 3 & 312 & -45.32 & -45.12 \\
\cline { 2 - 5 } & 5 & \multicolumn{2}{|c}{} \\
\hline
\end{tabular}

(a)
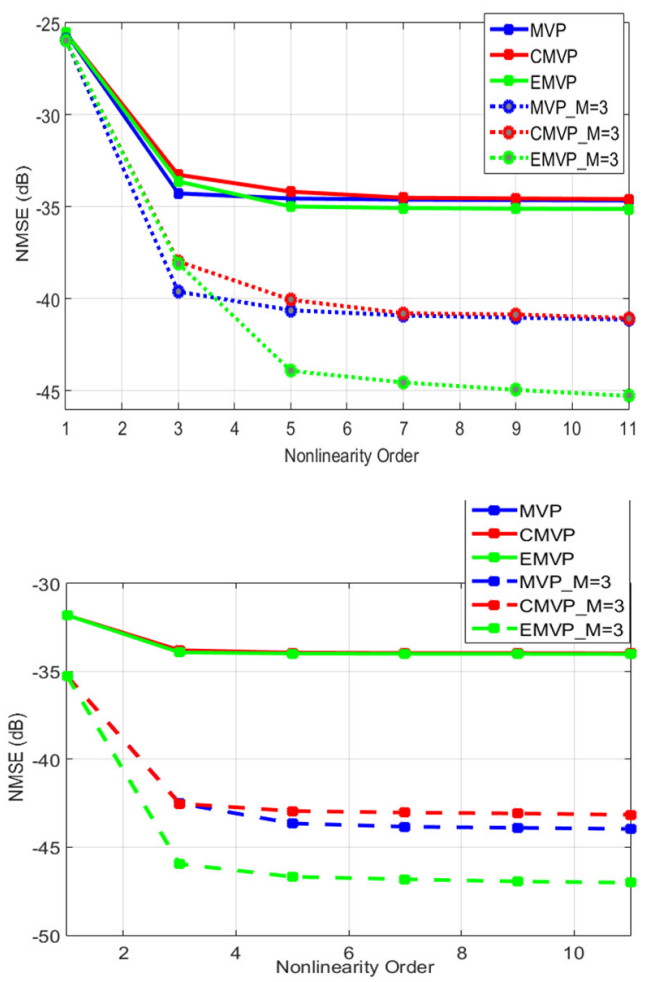

Fig. 4. The NMSE for surfaces as a function of the $\mathrm{K}$ and $\mathrm{M}$ values for different model, (a) scenario 1 (b) scenario 2

\section{2) DPD Results}

After evaluating the performance of the proposed multivariable polynomials in the reverse modeling, a DPD is built to linearize the MIMO transmitter. In this experiment, LC and NLC are set to $20 \mathrm{~dB}$ and the linearization experiment was performed two times, covering the two scenarios defined in Table III. Figure 5 presents the power spectral densities of the two output signals, with and without DPD, for the two coupling scenarios. The proposed DPD exhibited the lowest the highest linearization performance for both scenarios, which confirms the obtained ACPRs. Figures 5(c) and 5(d) show a small reduction in DPD's performance in the presence of linear coupling, even though the proposed DPD maintained its superiority over MVP and CMVP DPDs.
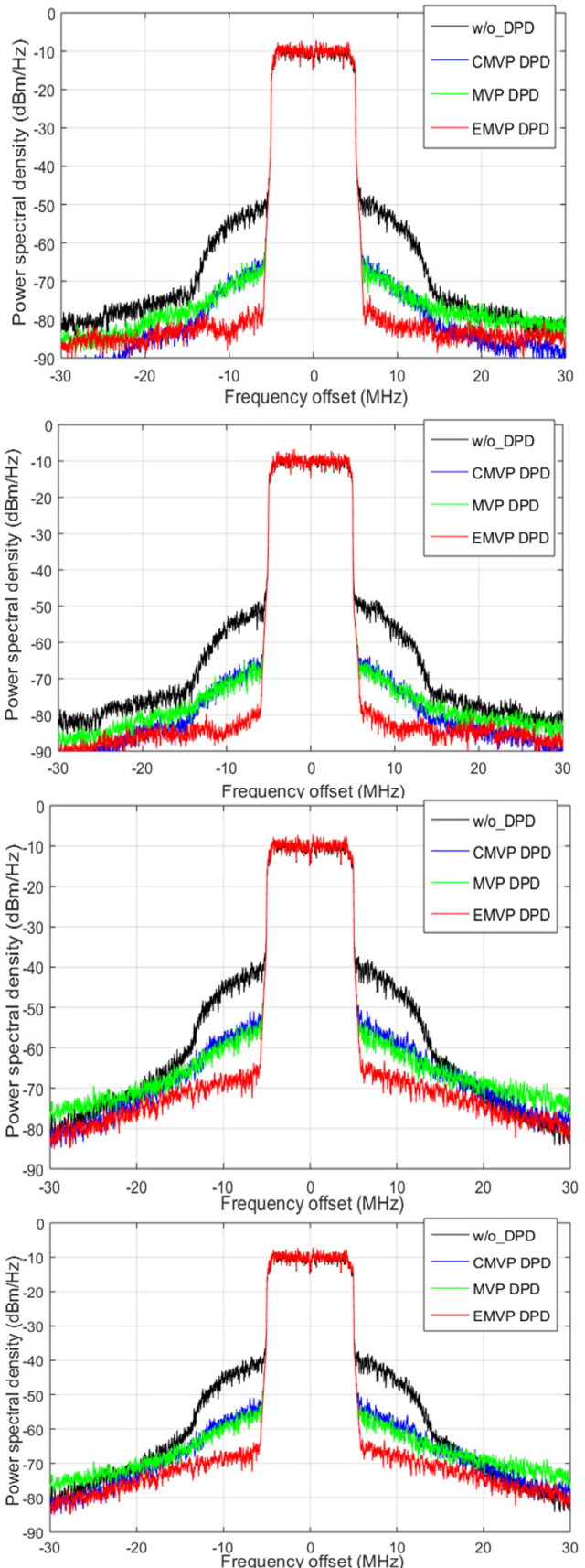

Fig. 5. Power spectral density (PSD) of the DUT output with and without DPD (a) channel 1, scenario 1, (b) channel 2, scenario 1, (c) channel 1, scenario 2, (d) channel 2, scenario 2 
TABLE VI. COMPARISON OF ACPR(DBC) FOR MIMO DPD TRANSMITTER WITH DIFFERENT MEASUREMENT SCENARIOS

\begin{tabular}{|c|c|c|c|c|}
\hline \multirow{2}{*}{ Models } & \multirow{2}{*}{ M } & \multirow{2}{*}{$\begin{array}{c}\text { Number of } \\
\text { coefficients }\end{array}$} & \multicolumn{2}{|c|}{ ACPR (dBc) } \\
\cline { 4 - 5 } & & Scenario 1 & $\boldsymbol{y}^{(2)}$ \\
\hline MVP & 5 & 792 & -52.83 & -52.23 \\
\hline CMVP & 5 & 312 & -50.74 & -51.74 \\
\hline EMVP & 3 & 208 & -63.02 & -62.02 \\
\hline \multicolumn{5}{|c|}{ Scenario 2 } \\
\hline MVP & 5 & 792 & -51.83 & -51.53 \\
\hline CMVP & 5 & 312 & -50.75 & -51.10 \\
\hline EMVP & 3 & 208 & -62.05 & -61.25 \\
\hline
\end{tabular}

\section{CONCLUSION}

This paper proposed a new behavioral memory polynomial model and a digital predistorter for $2 \times 2$ MIMO transmitters. This model includes nonlinear and cross terms, in order to reach a good compromise between efficiency and complexity. Robustness and stability of the model parameter identification procedure have been numerically validated for different nonlinearity orders and number of memory branches. The choice of polynomial cross terms has led to a good DPD performance, in the presence of strong linear and nonlinear crosstalk. The proposed MIMO DPD offers very competitive performance, compared to other recent publications, with no additional computational or hardware complexity.

\section{REFERENCES}

[1] J. Gozalvez, "Samsung electronics sets $5 \mathrm{G}$ speed record at $7.5 \mathrm{~Gb} / \mathrm{s}$ [mobile radio]", IEEE Vehicular Technology Magazine, Vol. 10, No. 1, pp. 12-16, 2015

[2] P. Pirinen, "A brief overview of 5G research activities", 1st International Conference on $5 \mathrm{G}$ for Ubiquitous Connectivity, Akaslompolo, Finland, November 26-28, 2014

[3] E. G. Larsson, O. Edfors, F. Tufvesson, T. L. Marzetta, "Massive MIMO for next generation wireless systems", IEEE Communications Magazine, Vol. 52, No. 2, pp. 186-195, 2014

[4] H. Q. Ngo, E. G. Larsson, T. L. Marzetta, "Energy and spectral efficiency of very large multiuser MIMO systems", IEEE Transactions on Communications, Vol. 61, No 4, pp. 1436-1449, 2013

[5] F. M. Ghannouchi, O. Hammi, M. Helaoui, Behavioral Modeling and Predistortion of Wideband Wireless Transmitters, John Wiley \& Sons, 2015

[6] M. Alizadeh, S. Amin, D. Ronnow, "Measurement and analysis of frequency-domain volterra kernels of nonlinear dynamic $3 \times 3$ MIMO systems", IEEE Transactions on Instrumentation and Measurement, Vol. 66, No. 7, pp. 1893-1905, 2017

[7] A. Vaezi, A. Abdipour, A. Mohammadi, F. M. Ghannouchi, "On the modeling and compensation of backward crosstalk in MIMO transmitters", IEEE Microwave and Wireless Components Letters, Vol. 27, No. 9, pp. 842-844, 2017

[8] A. Mohammadi, F. M. Ghannouchi, RF Transceiver Design for MIMO Wireless Communications, Springer, 2012

[9] S. A. Bassam, M. Helaoui, F. M. Ghannouchi, "Crossover digital predistorter for the compensation of crosstalk and nonlinearity in MIMO transmitters", IEEE Transactions on Microwave Theory and Techniques, Vol. 57, No. 5, pp. 1119-1128, 2009

[10] D. Saffar, N. Boulejfen, F. Ghannouchi, A. Gharsallah, M. Helaoui: "Behavioral modeling of MIMO nonlinear systems with multivariable polynomials", IEEE Transactions on Microwave Theory and Techniques, Vol. 59, No. 11, pp. 2994-3003, 2011

[11] P. Jaraut, M. Rawat, F. M. Ghannouchi, "Curtailed digital predistortion model for crosstalk in MIMO transmitters", 2018 IEEE/MTT-S
International Microwave Symposium - IMS, Philadelphia, USA, June $10-15,2018$

[12] A. Abdelhafiz, L. Behjat, F. M. Ghannouchi, M. Helaoui, O. Hammi, "A high-performance complexity reduced behavioral model and digital predistorter for MIMO systems with crosstalk", IEEE Transactions on Communications, Vol. 64, No. 5, pp. 1996-2004, 2016

[13] M. Younes, F. M. Ghannouchi, "Behavioral modeling of concurrent dual-band transmitters based on radially-pruned volterra model", IEEE Communications Letters, Vol. 19, No. 5, pp. 751-754, 2015

[14] E. Zenteno, D. Ronnow, "MIMO subband volterra digital predistortion for concurrent aggregated carrier communications", IEEE Transactions on Microwave Theory and Techniques, Vol. 65, No. 3, pp. 967-979, 2017

[15] N. Naraharisetti, P. Roblin, C. Quindroit, S. Gheitanchi, "Efficient leastsquares 2-D-cubic spline for concurrent dual-band systems", IEEE Transactions on Microwave Theory and Techniques, Vol. 63, No. 7, pp. 2199-2210, 2015

[16] Y. Liu, J. J. Yan, P. M. Asbeck, "Concurrent dual-band digital predistortion with a single feedback loop", IEEE Transactions on Microwave Theory and Techniques, Vol. 63, No. 5, pp. 1556-1568, 2015

[17] S. A. Bassam, A. Kwan, W. Chen, M. Helaoui, F. M. Ghannouchi, "Subsampling feedback loop applicable to concurrent dual-band linearization architecture", IEEE Transactions on Microwave Theory and Techniques, Vol. 60, No. 6, pp. 1990-1999, 2012

[18] S. A. Bassam, M. Helaoui, F. M. Ghannouchi, "2-D digital predistortion (2-D-DPD) architecture for concurrent dual-band transmitters", IEEE Transactions on Microwave Theory and Techniques, Vol. 59, No. 10, pp. 2547-2553, 2011

[19] H. Qian, X. Cheng, X. Luo, W. Feng, "Calibration of nonlinear crosstalk in MIMO transmitter", IEEE Transactions on Vehicular Technology, Vol. 66, No. 5, pp. 3739-3748, 2017

[20] A. K. Kwan, M. Younes, O. Hammi, M. Helaoui, N. Boulejfen, F. M. Ghannouchi, "Selective intermodulation compensation in a multi-stage digital predistorter for nonlinear multi-band power amplifiers", IEEE Journal on Emerging and Selected Topics in Circuits and Systems, Vol. 7, No. 4, pp. 534-546, 2017 